\title{
Heavy flavour production at LHCb
}

\author{
Marcello Rotondo*† \\ INFN Laboratori Nazionali di Frascati, Frascati, Italy \\ E-mail: marcello.rotondo@lnfn.infn.it
}

The measurements of heavy flavour production in $p p$ collisions provide crucial inputs to study both perturbative and non-perturbative quantum chromodynamics. Moreover these measurements allow to improve the precision on the study of heavy hadron decays. LHCb has a large program of studies of heavy flavour production and it has, compared with other experiments at the LHC, the unique feature to cover the very forward region. In this paper the most recent results on the quarkonia production and fragmentation fractions for various kinds of heavy hadrons are presented.

18th International Conference on B-Physics at Frontier Machines - Beauty2019 -

29 September / 4 October, 2019

Ljubljana, Slovenia

* Speaker.

${ }^{\dagger}$ On behalf of the LHCb collaboration 


\section{Introduction}

The detailed study of heavy-flavour production provides a crucial testing ground for both perturbative and non-perturbative aspects of quantum chromodynamics (QCD) calculations. Various models that predict the kinematics of heavy hadrons in a wide range of transverse momentum and pseudorapidy are available. Even if these models predict many features of the production, there are still many aspects of the mechanisms that require further studies from both the experimental and theoretical side.

In addition to the understanding of the underlying mechanism for heavy hadron formation, precise knowledge of the properties and kinematics of the heavy hadrons are crucial for the precise studies of their decays. For example the knowledge of the polarization of the $\Lambda_{b}$ in production is important for pentaquark analysis using $\Lambda_{b} \rightarrow J / \psi K^{-} p$ decays [1].

The LHCb detector [2,3], with its high momentum resolution, great particle identification capability and flexible trigger strategy, is perfectly suited to study heavy flavour production features. In this paper the following recent $\mathrm{LHCb}$ results on heavy hadron production are summarized:

- $\psi(2 S)$ production cross-sections [4],

- $\eta_{c}(1 S)$ production cross-section [5],

- $\Xi_{c c}^{++}$production [6],

- $B_{s}$ and $\Lambda_{b}$ production fractions [7],

- $B_{c}$ production fractions [8],

- $\Xi_{b}^{-}$production rate [9].

The datasets and the integrated luminosity used to obtain these results are reported in Table 1.

\section{Heavy quarkonium}

The study of heavy quarkonium production plays a central role because it helps in understanding how quarks combine into bound states. Heavy quarkonium production involves two steps: the production of a heavy quark pair, $q \bar{q}$, and its following hadronization in a specific quarkonium state.

Table 1: List of the analyses included in this paper with the datasets and the corresponding integrated luminosity used.

\begin{tabular}{cl|ccc}
\hline Reference & & $7 \mathrm{TeV}$ & $8 \mathrm{TeV}$ & $13 \mathrm{TeV}$ \\
\hline$[4]$ & $\psi(2 S)$ production cross-sections & $614 \mathrm{pb}^{-1}$ & - & $275 \mathrm{pb}^{-1}$ \\
{$[5]$} & $\eta_{c}(1 S)$ production cross-section & - & - & $2.0 \mathrm{fb}^{-1}$ \\
\hline$[6]$ & $\Xi_{c c}^{++}$production & - & - & $1.7 \mathrm{fb}^{-1}$ \\
{$[7]$} & $B_{s}$ and $\Lambda_{b}$ production fractions & - & - & $1.67 \mathrm{fb}^{-1}$ \\
{$[8]$} & $B_{c}$ production fractions & $1.0 \mathrm{fb}^{-1}$ & - & $1.7 \mathrm{fb}^{-1}$ \\
{$[9]$} & $\Xi_{b}^{-}$production rate & $1.0 \mathrm{fb}^{-1}$ & $1.0 \mathrm{fb}^{-1}$ & $1.6 \mathrm{fb}^{-1}$ \\
\hline
\end{tabular}


In general the first step can be computed with perturbative QCD, while the hadronization is merely non-perturbative and must be determined using experimental results. For this reason the underlying physics process of heavy quarkonium can probe both the perturbative and non-perturbative regime of QCD.

The nonrelativistic QCD approach [10] (NRQCD) predicts many features of quarkonium production and it is widely applicable. In this effective theory, intermediate $q \bar{q}$ states with all possible colour and spin quantum numbers, have probabilities to fragment into a specific quarkonium. The transition probabilities are described by long distance matrix elements (LDME) that must be determined from data. These matrix elements are assumed to be universal and can be applied to different quarkonium states. Despite the success of NRQCD in the description of quarkonium cross sections, it predicts a large transverse polarization of the $J / \psi$ that is not supported by experimental results. In the following, recent studies of the production of the $\psi(2 S)$ and $\eta_{c}$ states are reported.

\section{1 $\psi(2 S)$ production at $\sqrt{s}=7$ and $13 \mathrm{TeV}$}

In high energy $p p$ collisions, quarkonium states are produced from three different sources: directly from the hard collision, through feed-down of higher excited states or through the weak decays of $b$-hadrons. While the latter contribution can be disentangled exploiting the large lifetime of the $b$-hadrons, the contamination from the decays of excited states makes the comparison with the predictions complicated. For example the prompt $J / \psi$ production is affected by large feed-down from radiative decays of $\chi_{c}$ states. Instead the feed-down to the $\psi(2 S)$ state is almost negligible so theoretical predictions can be directly compared with the experimental results.

In this analysis the $\psi(2 S)$ production cross section is studied for both the prompt contribution, prompt $\psi(2 S)$, and from $b$-hadron decays, $\psi(2 S)$-from- $b$. The $\psi(2 S)$ are reconstructed through the $\psi(2 S) \rightarrow \mu^{+} \mu^{-}$decay mode. The kinematic region in rapidity and transverse momentum analysed is $2.0<y<4.5$, and $3.5<p_{T}<14 \mathrm{GeV} / \mathrm{c}$ at $7 \mathrm{TeV}$, and $2<p_{T}<14 \mathrm{GeV} / \mathrm{c}$ at $13 \mathrm{TeV}$. The variable most useful to separate prompt $\psi(2 S)$ and $\psi(2 S)$ from $b$-decays is the pseudo decay time $t_{z}$, defined as

$$
t_{z}=\left(z_{\psi(2 S)}-z_{P V}\right) \cdot \frac{M_{\psi(2 S)}}{p_{z}},
$$

where $z_{\psi(2 S)}$ and $z_{P V}$ are the $z$-coordinates of the reconstructed $\psi(2 S)$ decay vertex and the associated Primary Vertex (PV), respectively; $p_{z}$ is the $z$-component of the measured $\psi(2 S)$ momentum, and $M_{\psi(2 S)}$ is the $\psi(2 S)$ mass.

The main result of the analysis is the differential cross section $d^{2} \sigma / d y d p_{T}$ separately for the prompt $\psi(2 S)$ and $\psi(2 S)$-from- $b$ samples. The differential cross section in a given $\left(y, p_{T}\right)$ bin, is obtained from the $\psi(2 S)$ yields, corrected for the total efficiency to reconstruct a $\psi(2 S)$, the integrated luminosity and the $\psi(2 S) \rightarrow \mu^{+} \mu^{-}$branching fraction. The yields of prompt $\psi(2 S)$ and $\psi(2 S)$ from $b$-decay candidates are determined in each $\left(y, p_{T}\right)$ bin by a two dimensional fit to the distribution of the invariant mass $m_{\mu^{+} \mu^{-}}$and the pseudo-proper time $t_{z}$. In Fig. 1 is shown an example of the $m_{\mu^{+} \mu^{-}}$and $t_{z}$ distributions in a single bin of $y$ and $p_{T}$. The distribution of $t_{z}$ is described by a $\delta$ function for the prompt $\psi(2 S)$ and an exponential for the $\psi(2 S)$-from- $b$, both convolved with an appropriate resolution function. The background is described by an empirical function whose shape is guided by the events in the $m_{\mu^{+} \mu^{-}}$side bands. 

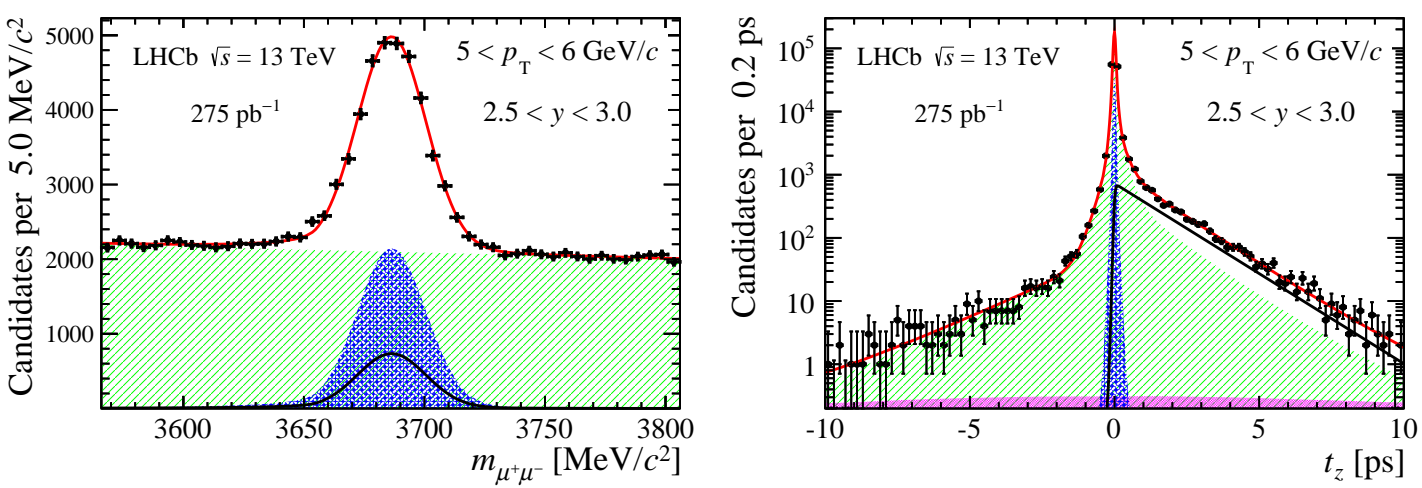

Figure 1: Distributions of the invariant mass $m_{\mu^{+} \mu^{-}}$(left) and the pseudo decay time $t_{z}$ (right) of selected $\psi(2 S)$ candidates in a bin of $y$ and $p_{T}$ in the $13 \mathrm{TeV}$ data sample. Projections of the two-dimensional fit result are also shown.

The main result is the double-differential production cross-section $d^{2} \sigma / d y d p_{T}$ for prompt $\psi(2 S)$ and $\psi(2 S)$-from- $b$, assuming no polarisation of the $\psi(2 S)$. The distributions of $d \sigma / d p_{T}$, obtained integrating the double-differential cross section over $y$, are shown in Fig.2 for both data at $7 \mathrm{TeV}$ and $13 \mathrm{TeV}$. The predictions for the prompt $\psi(2 \mathrm{~S})$ production based on the NRQCD [10], and for the $\psi(2 S)$-from- $b$ obtained with the Fixed Order plus Next-to-Leading Logarithm (FONLL) calculations [11], are superimposed on the experimental results. The NRQCD calculation shows reasonable agreement with the data for $p_{T}>7 \mathrm{GeV} / c$, while predictions are omitted at lower momentum where they are not reliable. The FONLL calculation agrees very well with the measurements in the full range considered. The same good agreement is present for the $y$ distributions obtained by integrating over $p_{T}$.

The production cross sections of $\psi(2 S)$ are compared with those of the $J / \psi$ mesons measured by LHCb at $\sqrt{s}=13 \mathrm{TeV}$ [12]. These ratios of cross sections, $R(\psi(2 S) / J / \psi)$, increase with $p_{T}$ for both prompt and from- $b \psi(2 S)$. The FONLL calculation predicts very well the ratio $R(\psi(2 S) / J / \psi)$ for $\psi(2 S)$ and $J / \psi$ from $b$-hadron decays, while the NRQCD shows only reasonable agreement for prompt $\psi(2 S)$ and $J / \psi$.

The production cross-sections of the $\psi(2 S)$ are also compared for the $13 \mathrm{TeV}$ and $7 \mathrm{TeV}$ samples. The ratio $R_{13 / 7}$ is about 1.5 for prompt $\psi(2 S)$ and it increases slightly with the $p_{T}$; this behaviour is well predicted by the NRQCD model. For the $\psi(2 S)$-from- $b$ the measured ratio $R_{13 / 7}$ is well predicted by the FONLL calculation.

\section{2 $\eta_{c}(1 S)$ production at $\sqrt{s}=13 \mathrm{TeV}$}

The NRQCD framework allows to simultaneously treat the $J / \psi\left(J^{C P}=1^{--}\right)$and $\eta_{c}\left(J^{C P}=\right.$ $0^{-+}$) production imposing relations between their LDME, under the assumption of factorization and heavy-quark spin symmetry. The simultaneous study of $\eta_{c}$ and $J / \psi$ prompt production and production from $b$-hadron decays, provides an important test of the basic NRQCD assumptions.

In this analysis the differential production cross-section of the $\eta_{c}$ is measured in the region $6.5<p_{T}<14.0 \mathrm{GeV} / c$ and $2.0<y<4.5$. The measurement is performed relative to that of the $J / \psi$ meson, in four bins of $p_{T}$. Both the $\eta_{c}$ and the $J / \psi$ are reconstructed in the $p \bar{p}$ decay mode. 

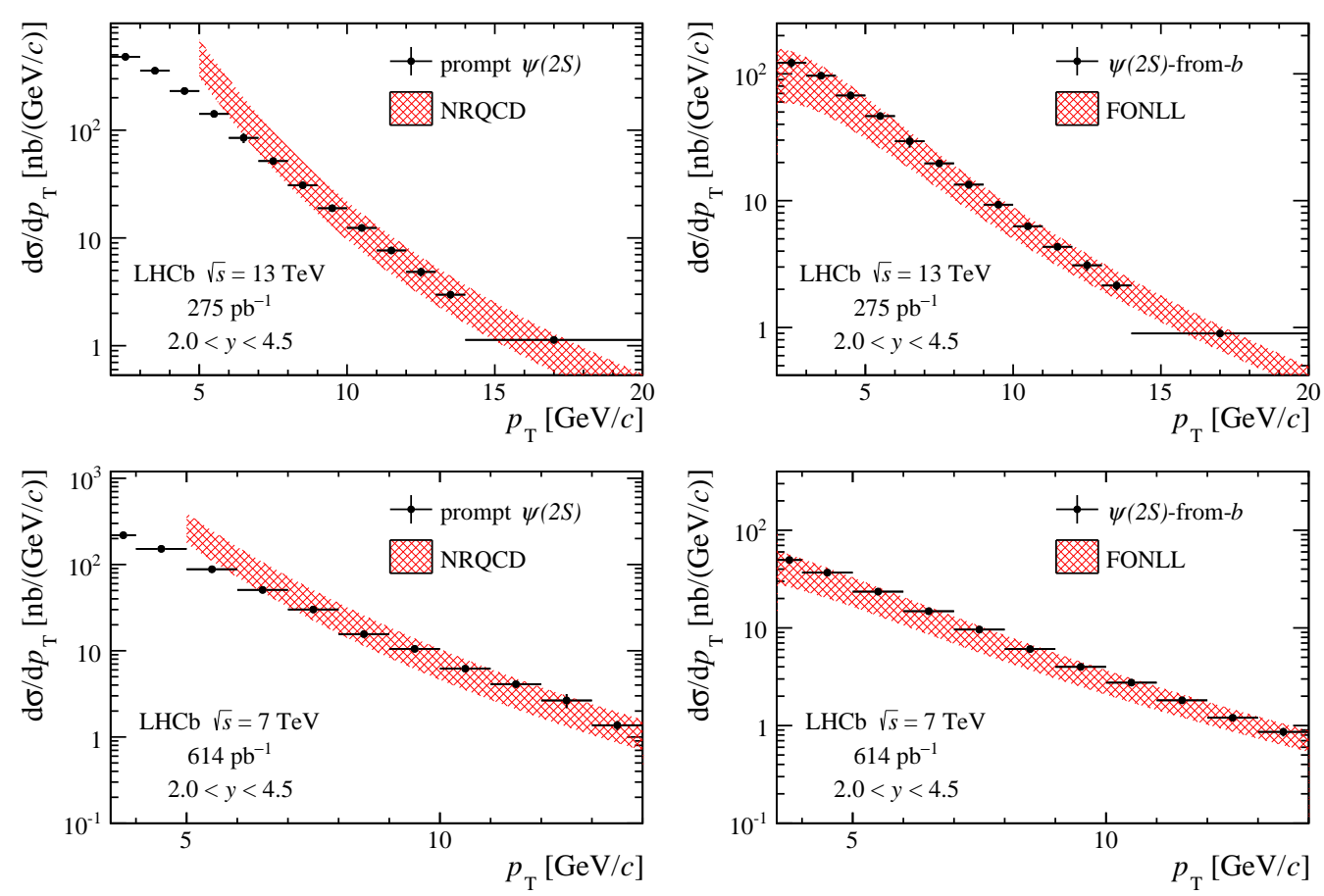

Figure 2: Differential production cross-sections as functions of $p_{T}$ for the $13 \mathrm{TeV}$ (top) and the $7 \mathrm{TeV}$ sample (bottom). The figures on the left are for prompt $\psi(2 S)$ and the results compared with the NRQCD [10] calculations; the figures on the right are for $\psi(2 S)$-from- $b$ and the results are compared with the FONLL calculations [11].

The actual measurements are the ratios of cross sections, $\left(d \sigma_{\eta_{c}} / d p_{T}\right) /\left(d \sigma_{J / P s i} / d p_{T}\right)$, separately for prompt $\eta_{c}$ and $\eta_{c}$-from- $b$ decays. Because the $p \bar{p}$ pairs from $\eta_{c}$ and $J / \psi$ decays have comparable kinematics, they have similar reconstruction, trigger and selection efficiencies. The prompt $\eta_{c}$ and $J / \psi$ are distinguished from the $b$-hadron decays using the pseudo-proper time $t_{z}$ defined as in Eq.2.1. A prompt-enriched sample is defined requiring $t_{z}<80 \mathrm{fs}$, while a $b$-hadron enriched sample is selected by $t_{z}>80 \mathrm{fs}$. The small cross-feed between the two samples is evaluated with simulation.

The $J / \psi$ yields and the ratio of $\eta_{c}$ and $J / \psi$ are extracted from a simultaneous fit to the $p \bar{p}$ invariant mass distribution in the prompt-enriched and $b$-hadron enriched samples. The $p \bar{p}$ invariant mass distributions are shown in Fig. 3 with the result of the fits, integrated over $p_{T}$, superimposed.

The ratio of prompt production of $\eta_{c}$ and $J / \psi$ as a function of $p_{T}$ is shown in Fig.4 (left). After integration over $p_{T}$, the relative prompt production cross section $\sigma_{\eta_{c}} / \sigma_{J / \psi}$ is $1.69 \pm 0.15_{\text {stat }} \pm$ $0.10_{\text {syst }} \pm 0.18_{B F}$ where the last uncertainty is due to the $J / \psi \rightarrow p \bar{p}$ and $\eta_{c} \rightarrow p \bar{p}$ branching fractions. This measurement supports the conclusions of the LHCb measurement at $\sqrt{s}=7$ and $8 \mathrm{TeV}$, reported in [13], that $\eta_{c}$ production is enhanced compared to that of the $J / \psi$. Using $\sigma_{J \psi}=0.749 \pm 0.047 \mu b$ for the prompt $J / \psi$ production [12], the prompt $\eta_{c}$ production cross section in the considered fiducial region is

$$
\sigma_{\eta_{c}}^{\text {prompt }}=1.26 \pm 0.11_{\text {stat }} \pm 0.08_{\text {syst }} \pm 0.14_{\sigma_{J / \psi}} \mu b,
$$

which is in good agreement with the colour-singlet model prediction, $1.56_{-0.49-0.17}^{+0.83+0.38} \mu b$ [14]. The 

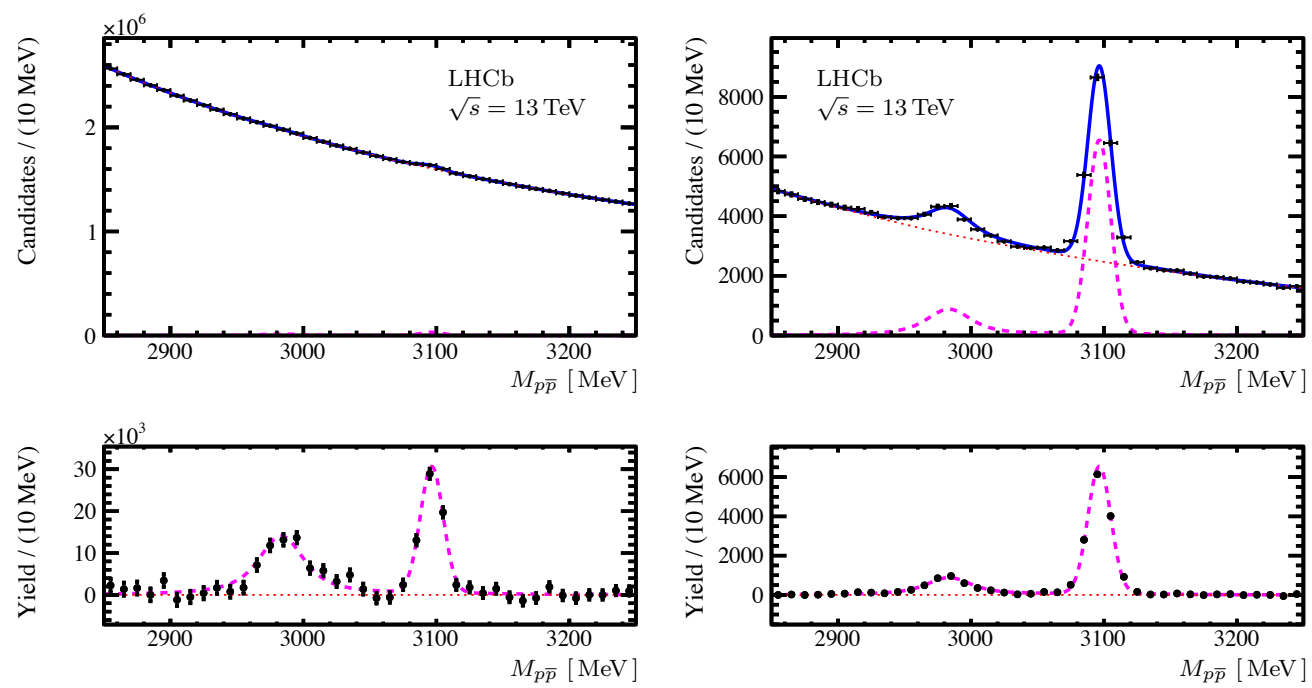

Figure 3: Invariant-mass distribution of the $p p$ candidates for (top left) prompt-enriched with $t_{z}<80 \mathrm{fs}$ and (top right) $b$-hadron enriched with $t_{z}>80 \mathrm{fs}$ samples. Signal distributions with the background components from the fit subtracted are shown on the bottom plots for the two samples.
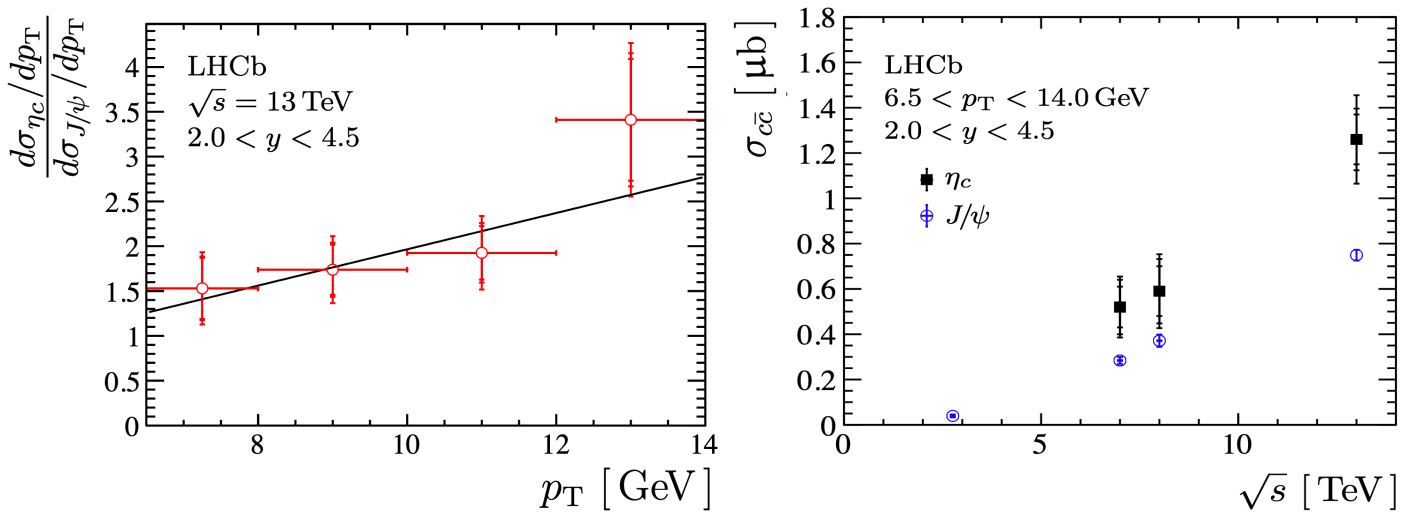

Figure 4: (Left) Ratios of $\eta_{c}$ to $J / \psi$ differential production cross-sections for prompt production. The result of a fit with a linear function is overlaid. (Right) Absolute $\eta_{c}$ (black rectangles) and $J / \psi$ (blue circles) prompt production cross-sections.

LHCb result confirms the theoretical analyses $[15,16,17]$ that follow the LHCb first measurements at $\sqrt{s}=7$ and $8 \mathrm{TeV}$. It is worth mentioning that these theoretical analyses do not predict the slope observed in Fig.4(left) that shows $p_{T}$ dependent differences between the $J / \psi$ and $\eta_{c}$ production cross sections. Further studies are needed, for example extending the $p_{T}$ region analysed.

The $\eta_{c}$ production cross-section dependence on $\sqrt{s}$ is shown in Fig.4 (right) with the results on the prompt $J / \psi$ cross section superimposed for reference. The relative $\eta_{c}$ production rate from $b$-hadron decays is measured to be $\mathscr{B}\left(b \rightarrow \eta_{c} X\right) / \mathscr{B}(b \rightarrow J / \psi X)=0.48_{\text {stat }} \pm 0.03_{\text {syst }} \pm 0.05_{B F}$, which is the most precise measurement of the inclusive $b \rightarrow \eta_{c} X$ branching ratio and is in good agreement with a previous LHCb measurement [13]. 


\section{Open charm and beauty}

The measurements of branching fractions of specific decay channels of $b$-hadrons are often needed to make quantitative comparisons with theoretical predictions. The absolute branching fraction measurements at hadron colliders are difficult without external inputs. In general the branching fraction of a decay channel is measured relative to a channel with similar decay topology, that often is a decay of a $B^{0}$ or $B^{-}$meson whose absolute branching ratio is known well from B-factories. These measurements require the knowledge of the production fraction of any specific heavy hadron $H_{b}$, called $f_{H_{b}}$, which is the probability that a $b$-quark fragments into the $H_{b}$ hadron. For example the probabilities that a $b$-quark hadronize into a $B^{0}, B_{s}^{0}, \Lambda_{b}^{0}$ or a $B_{c}^{+}$, are $f_{d}, f_{s}, f_{\Lambda_{b}}$ and $f_{c}$ respectively.

\section{1 $\Xi_{c c}$ production at $\sqrt{s}=13 \mathrm{TeV}$}

Since the observation of the $\Xi_{c c}^{++}$baryon into the $\Lambda_{c}^{+} K^{-} \pi^{+} \pi^{+}$decay mode [18], many studies have been published to study its property: the lifetime has been measured and found consistent with a weak decay, the decay mode $\Xi_{c c}^{++} \rightarrow \Xi_{c}^{+} \pi^{+}$has been observed, and a search for the single charmed decay $\Xi_{c c}^{++} \rightarrow D^{+} p K^{-} \pi^{+}$has found no signal. The data sample corresponding to $1.5 \mathrm{fb}^{-1}$ taken at $\sqrt{s}=13 \mathrm{TeV}$ is used to study $\Xi_{c c}^{++}$production. In this measurement the $\Xi_{c c}^{++}$production cross section times its branching fraction into $\Lambda_{c}^{+} K^{-} \pi^{+} \pi^{+}, \sigma\left(\Xi_{c c}^{++}\right) \times \mathscr{B}\left(\Xi_{c c}^{++} \rightarrow \Lambda_{c}^{+} K^{-} \pi^{+} \pi^{+}\right)$, is measured relative to the $\Lambda_{c}^{+}$prompt production cross section, $\sigma\left(\Lambda_{c}^{+}\right)$, in the kinematic region $4<p_{T}<15 \mathrm{GeV} / c$ and $2.0<y<4.5$.

The $\Lambda_{c}^{+}$, reconstructed in the $p K^{-} \pi^{+}$decay mode, is required to form a good vertex wellseparated from the PV. The additional tracks have to be well identified, and have to form a vertex with the $\Lambda_{c}^{+}$candidate. The formed $\Xi_{c c}^{++}$candidates are required to have a $p_{T}$ greater than $4 \mathrm{GeV}$ and to originate from a PV. The combinatorial background is suppressed using a multivariate classifier using vertex and kinematic information of the $\Xi_{c c}^{++}$decay products.

After all the selection requirements, the distribution of the invariant mass of $\Xi_{c c}^{++}$candidates is shown in Fig. 5 for two different trigger lines used.
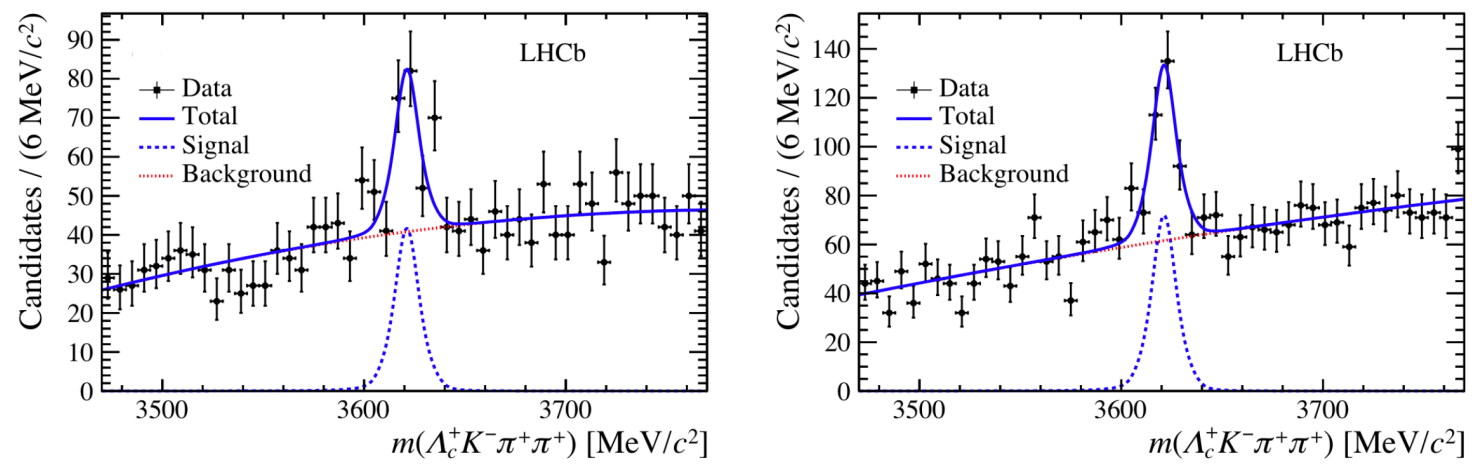

Figure 5: Invariant-mass distributions of $\Xi_{c c}^{++}$candidates (left) triggered by particles belonging to the signal and (right) triggered exclusively by particles unrelated to the signal.

The prompt $\Lambda_{c}^{+}$production sample requires particular care, because of the contamination from $\Lambda_{c}^{+}$'s from $b$-hadron decays. A powerful variable useful to disentangle these two source of $\Lambda_{c}^{+}$is 
the $\Delta \chi_{I P}$ which is defined as the difference in the $\chi^{2}$ of the PV fit with and without the particles from the $\Lambda_{c}^{+}$decay candidate. The prompt $\Lambda_{c}^{+}$are characterized by lower values of $\Delta \chi_{I P}^{2}$, while $\Lambda_{c}^{+}$ from other processes have larger $\Delta \chi_{I P}$ values. The efficiency to reconstruct $\Xi_{c c}^{++}$'s depends strongly on $\Xi_{c c}^{++}$lifetime, and assuming the measured central value [19], the resulting $\Xi_{c c}^{++}$production cross section ratio is

$$
\frac{\sigma\left(\Xi_{c c}^{++}\right) \times \mathscr{B}\left(\Xi_{c c}^{++} \rightarrow \Lambda_{c}^{+} K^{-} \pi^{+} \pi^{-}\right)}{\sigma\left(\Lambda_{c}^{+}\right)}=\left(2.22 \pm 0.27_{\text {stat }} \pm 0.29_{\text {syst }}\right) \times 10^{-4} .
$$

This is the first measurement of the production of a doubly charmed baryon in $p p$ collisions and gives important input to improve our understanding on its production mechanism.

\section{2 $\Lambda_{b}$ and $B_{s}$ production at $\sqrt{s}=13 \mathrm{TeV}$}

In this analysis the ratios $f_{s} /\left(f_{u}+f_{d}\right)$ and $f_{\Lambda_{b}^{0}} /\left(f_{u}+f_{d}\right)$ are extracted in the pseudorapidity range $2<\eta<5$ and transverse momentum range $4<p_{T}<25 \mathrm{GeV} / \mathrm{c}$ using the data at $\sqrt{s}=$ $13 \mathrm{TeV}$. Because these ratios can depend on $p_{T}$ and $\eta$, a two- dimensional fit is performed in these variables.

This measurement exploits the theoretical prediction that the inclusive semileptonic decay widths, $\Gamma_{S L}$, are nearly the same for all $b$-hadrons with light or strange quark spectators [20]. As a consequence, the inclusive semileptonic branching ratio for the $B_{s}$ and the $\Lambda_{b}$ can be related to the precisely measured inclusive branching ratio $\mathscr{B}\left(B \rightarrow X_{c} \ell v_{\ell}\right)=\tau_{B} \Gamma_{S L}\left(B^{0}+B^{+}\right)=(10.70 \pm 0.19) \%$ obtained at the B-Factories [21] through the following relations,

$$
\begin{aligned}
\mathscr{B}\left(B_{s} \rightarrow X_{c}\right) & =\tau_{s}\left(\Gamma_{S L}(B)+\eta_{s}\right), \\
\mathscr{B}\left(\Lambda_{b}^{0} \rightarrow X_{c}\right) & =\tau_{\Lambda_{b}}\left(\Gamma_{S L}(B)+\eta_{\Lambda_{b}}\right),
\end{aligned}
$$

where $\tau_{s}$ and $\tau_{\Lambda_{b}}$ are the known lifetimes of $B_{s}$ mesons and $\Lambda_{b}$ baryons respectively, and $\eta_{s}$ and $\eta_{\Lambda_{b}}$ are small corrections to $\Gamma_{S L}$ for $B_{s}(-1.0 \pm 0.5) \%$ and $\Lambda_{b}(+3.0 \pm 1.5) \%$ taken from [20]. The yields of the inclusive semileptonic decays of $B^{0}$ and $B^{+}$are obtained as mixtures of decays $B^{0,+} \rightarrow$ $D^{0} \mu v X$ and $B^{0,+} \rightarrow D^{+} \mu v X$, properly corrected for the selection and reconstruction efficiencies, and for the presence of a small component of $B \rightarrow D_{s}^{(*)+} K \mu \nu$ decays that do not give $D^{+}$or $D^{0}$ in the final state. Similarly in the semileptonic decays of the $B_{s}$, mostly there is a $D_{s}^{+}$in the final state. However there is also a non-negligible contribution that gives $D^{0} K^{+}$and $D^{+} K^{0}$ pairs through excited $D_{s}^{* *}$ decays or from non-resonant $D K$ pairs. These contributions are evaluated on data reconstructing explicitly $D^{0} K^{+} \mu \nu X$ events. Analogously, the $\Lambda_{b}^{0}$ decays semileptonically mainly with a $\Lambda_{c}^{+}$in the final state, but there can also be $D^{0} p$ or $D^{+} n$ pairs produced. These are determined studying the $D^{0} p \mu \nu X$ sample.

The different combinations analysed are $D^{0} \mu \nu X, D^{+} \mu \nu X, D_{s}^{+} \mu \nu X$ and $\Lambda_{c}^{+} \mu \nu X$. In all cases, well reconstructed $D^{0} \rightarrow K \pi, D^{+} \rightarrow K 3 \pi, D_{s}^{+} \rightarrow K^{+} K^{-} \pi^{+}$and $\Lambda_{c}^{+} \rightarrow p K^{-} \pi^{+}$, are required to form a good vertex well separated from the PV and not pointing to the PV itself. These hadrons are combined with a well identified $\mu$ with large $p_{T}$ and coming from the same vertex.

The remaining component of $B_{s}^{0}$ decaying into $D K$ must be added into the $B_{s}$ yields and subtracted from the $B^{0}$ and $B^{+}$yields. For the $\Lambda_{b}$ the $D p$ component has to be added to the $\Lambda_{b}^{0}$ yields and subtracted from the fractions $f_{u}$ and $f_{d}$. These signal components are evaluated adding 

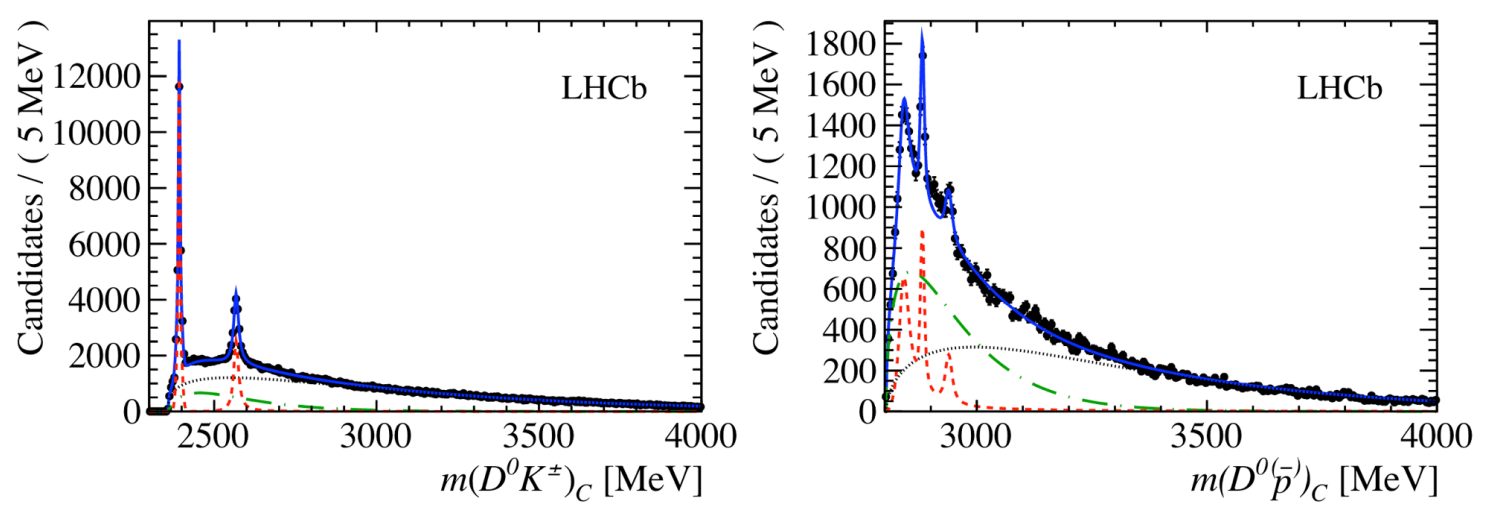

Figure 6: (Left) Projections of the two-dimensional fits onto the $m\left(D^{0} K^{+}\right)$for $D^{0} K^{+} \mu v X$ candidates (left). The two peaks correspond to the excited states $D_{s 1}(2536)^{+}$and $D_{s 2}^{*}(2537)^{+}$. The non-resonant component (long-dashed, green) corresponds to about $50 \%$ of the $D^{0} K^{+}$sample. (Right) Projection of the $m\left(D^{0} \bar{p}\right)$ mass distribution for $D^{0} p \mu v X$ candidates. The peaks due to $\Lambda_{c}^{+}(2860), \Lambda_{c}^{+}(2880)$ and $\Lambda_{c}^{+}(2940)$ are visible. The non-resonant component is about $76 \%$ of the $D^{0} p$ sample.

a further well identified $K$ or a proton, to the $D^{0} \mu \nu X$ samples. The resonant and non-resonant $B_{s}^{0} \rightarrow D^{0} K^{+} \mu v X$ and $\Lambda_{b}^{0} \rightarrow D^{0} p \mu v X$ components are isolated from the large combinatorial background, through a two-dimensional fit to the distribution of the constrained $D K$ invariant mass, defined as $m(D K)_{C}=m(D K)-m(D)+m(D)_{P D G}$ (analogously $m(D p)_{C}$ mass, with an identified proton), and the $\Delta \chi_{V}$, which is defined as the difference between the vertex $\chi^{2}$ formed by the added hadron track and the $D \mu$ system and the vertex $\chi^{2}$ of the $D \mu$ system. The variable $\Delta \chi_{V}$ is smaller for events where the $D K$ and $D p$ combinations come from resonant or non resonant $B_{s} \rightarrow D^{0} K^{+} \mu v_{\mu}$ and $\Lambda_{b} \rightarrow D^{0} p \mu v_{\mu}$ semileptonic decays, and it takes a larger value if the additional track (kaon or proton) is not associated with the $D \mu$ vertex. The projection of these fits on $m(D K)$ and $m(D p)$ distributions are shown in Fig.6 where, for both samples, the presence of excited states are clearly observed together with large non-resonant components. The unmeasured $D^{+} K^{0}$ and $D^{+} n$ are properly accounted using isospin conservation.

The production of the $b$-hadron $H_{b}\left(B_{s}^{0}\right.$ or $\left.\Lambda_{b}^{0}\right)$ is evaluated in bins of $\eta$ and $p_{T}\left(H_{b}\right)$. The pseudorapidity is determined by measuring the angle of the $H_{b}$ hadron, whose direction is determined from the PV and the $H_{b}$ decay vertex, with respect to the beam direction. Because of the presence of the neutrinos the $p_{T}\left(H_{b}\right)$ cannot be reconstructed directly. Instead $p_{T}\left(H_{b}\right)$ is inferred from the visible $H_{c} \mu$ systems applying a correction factor, $k$-factor, that is the ratio of the average reconstructed $p_{T}\left(H_{c} \mu\right)$ to the true $p_{T}\left(H_{b}\right)$, as a function of the invariant mass $m\left(H_{c} \mu\right)$. For illustration, the distribution of this ratio and the $k$-factor correction for the $B_{c} \rightarrow J / \psi \mu \nu$ decay mode is shown in Fig. 8 (right).

The distribution of $f_{s} /\left(f_{u}+f_{d}\right)$ and $f_{\Lambda_{b}} /\left(f_{u}+f_{d}\right)$ as a function of $p_{T}$ are shown in Fig.7 (left). The distribution of $f_{s} /\left(f_{u}+f_{d}\right)$ is fitted with a linear function and the slope is significantly different from zero. The ratio $f_{\Lambda_{b}} /\left(f_{u}+f_{d}\right)$ shows instead a large $p_{T}$ dependence, already observed in other $\mathrm{LHCb}$ analyses $[22,23]$. The average values of the two ratios, obtained by integration over the 

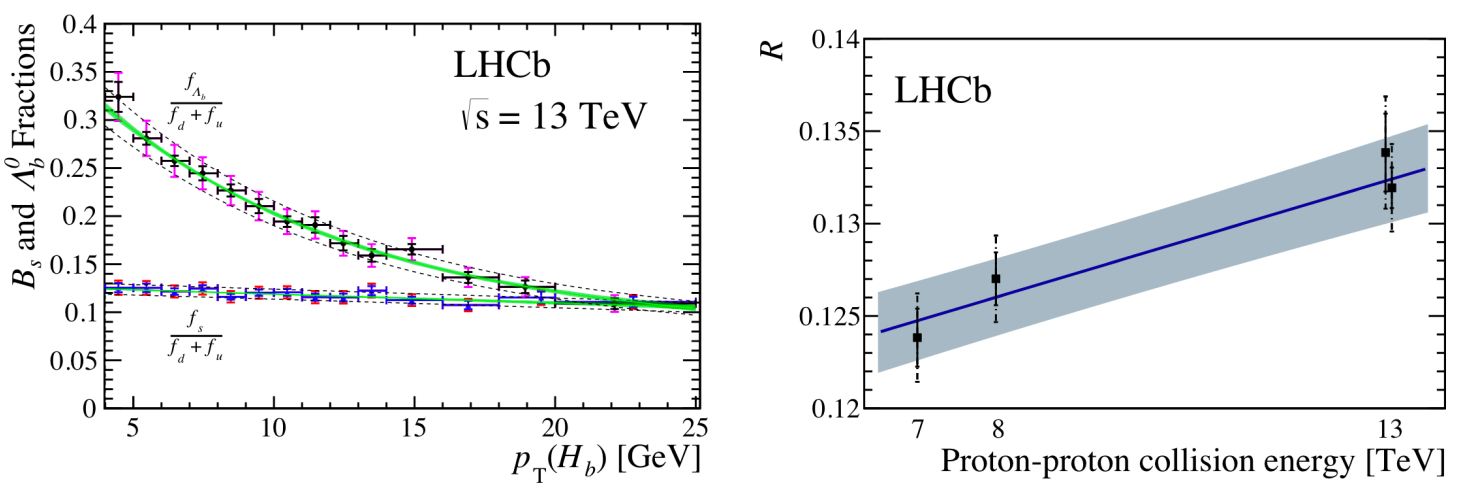

Figure 7: (Left) The $B_{s}$ and $\Lambda_{b}$ production fractions relative to $B^{0}+B^{+}$, in bins of $p_{T}\left(H_{b}\right)$. (Right) Efficiency-corrected $B_{s}^{0} \rightarrow J / \psi \phi$ and $B^{+} \rightarrow J / \psi K^{+}$yield ratios, $R$, at different $p p$ collision energies as reported from $\mathrm{LHCb}$ in [24]. The ratio $R$ is proportional to $f_{s} / f_{u}$.

kinematic range $4<p_{T}<25 \mathrm{GeV} / \mathrm{c}$ and $2<\eta<5$, are

$$
\frac{f_{s}}{f_{u}+f_{d}}=0.122 \pm 0.006, \quad \frac{f_{\Lambda_{b}^{0}}}{f_{u}+f_{d}}=0.259 \pm 0.018
$$

where the dominant sources of uncertainty are systematic and mainly due to external parameters. For $f_{s} /\left(f_{u}+f_{d}\right)$ the dominant systematic is the branching ratio of the decay $D_{s}^{+} \rightarrow K^{+} K^{-} \pi^{+}$ that gives a contribution of $3.3 \%$ to the systematic uncertainty. For $f_{\Lambda_{b}} /\left(f_{u}+f_{d}\right)$ the dominant uncertainty is due to the branching ratio of the $\Lambda_{c}^{+} \rightarrow p K^{+} \pi^{-}$decay, which gives a contribution of $5.3 \%$ to the total systematic.

The result obtained using $7 \mathrm{TeV}$ data [25], $f_{s} /\left(f_{u}+f_{d}\right)=0.1295 \pm 0.0075$, is consistent with the present result at $13 \mathrm{TeV}$. A recent LHCb measurement [24] studied the ratio $f_{s} / f_{u}$ using the efficiency-corrected ratio $(R)$ of yields between $B_{s}^{0} \rightarrow J / \psi \phi$ and $B^{+} \rightarrow J / \psi K^{+}$decays. The result confirms a dependence of $B_{s}^{0}$ production on $p_{T}\left(H_{b}\right)$, but also shows a significant dependence of $f_{s} / f_{u}$ with $\sqrt{s}$ as shown in Fig.7 (right). This requires further studies, in particular an analysis with semileptonic decays as presented here should be repeated with data at 7 and $8 \mathrm{TeV}$ using all the updated analysis strategies and external inputs.

\section{3 $B_{c}^{-}$production at $\sqrt{s}=13 \mathrm{TeV}$}

In this analysis, the $B_{c}^{-}$production fraction, $f_{c}$, is measured relative to the sum of $B^{0}$ and $B^{-}$, $f_{d}+f_{u}$, using a technique similar to the one used for $B_{s}^{0}$ and $\Lambda_{b}^{0}$ production. However, for the $B_{c}^{-}$, the inclusive semileptonic decay width is not equal to the other $b$-hadrons with light or strange quarks as in Eq.3.1, because both the $b$ and $c$ quarks can decay. Instead this analysis relies on the theoretical predictions of the branching fraction $\mathscr{B}\left(B_{c}^{-} \rightarrow J / \psi \mu^{-} v\right)$. The ratio $f_{c} /\left(f_{u}+f_{d}\right)$ is obtained as function of the transverse momentum of the $B_{c}$, and $\eta$.

The $B_{c}^{-} \rightarrow J / \psi \mu \nu$ events are selected from $J / \psi \rightarrow \mu^{+} \mu^{-}$candidates with good vertex quality and a large flight distance from the PV. The other muon must be well identified and has to form a good vertex with the $J / \psi$. The variable used to extract the signal yields is the corrected mass defined as $m_{\text {cor }}=\sqrt{m(J / \psi \mu)^{2}+p_{\perp}^{2}}+p_{\perp}$, where $p_{\perp}$ is the magnitude of the $J / \psi \mu$ momentum 
transverse to the $B_{c}^{-}$candidate direction of flight. The variable $m_{c o r}$ is useful to suppress the contamination from $B_{c}^{-} \rightarrow J / \psi \tau^{-} \bar{v}_{\tau}\left(\tau^{-} \rightarrow \mu^{-} v_{\tau} \bar{v}\right), B_{c}^{-} \rightarrow \psi(2 S) \mu^{-} \bar{v}$ and $B_{c}^{-} \rightarrow \chi_{c} \mu^{-} \bar{v}$ with the subsequent decay of the $\psi(2 S)$ and $\chi_{c}$ into a $J / \psi$. The shape of $m_{c o r}$ for the $13 \mathrm{TeV}$ data sample is shown in Fig. 8(left). There are about 4000 signal candidates in the $7 \mathrm{TeV}$ sample and about 15000 in the $13 \mathrm{TeV}$ sample.

The ratio $f_{c} /\left(f_{u}+f_{d}\right)$ can vary as function of $p_{T}$ and $\eta$ of the $B_{c}$, as observed in the $\Lambda_{b}^{0}$ and $B_{s}^{0}$ production. Hence the measurement is performed in bins of $\eta$ and $p_{T}$. Because of the presence of the neutrino, the $p_{T}$ is inferred using the $k$-factor as described in Section 3.2. The distribution of the ratio between the visible $p_{T}$ and the true one in simulation is shown in Fig.8 (right).
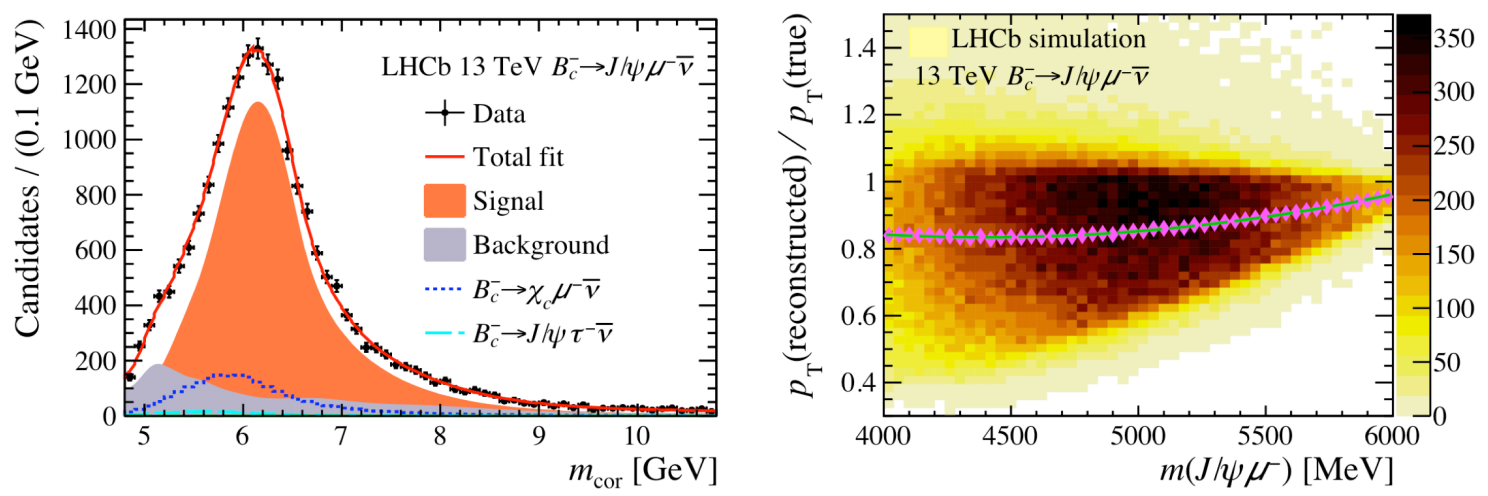

Figure 8: (Left) Fitted $m_{\text {cor }}$ distributions in the $13 \mathrm{TeV}$ sample. The signal and the backgrounds are shown as orange and grey areas. (Right) The k-factor corrections as a function of invariant mass of $m(J / \psi \mu)$ in simulation. The points (magenta) are the average k-factor corrections.

The ratio $f_{c} /\left(f_{u}+f_{d}\right)$ does not show significant dependence on pseudorapidity $\eta$ in the range $2.5<\eta<4.5$, as can be seen in Fig.9(left). Instead $f_{c} / f_{u}+f_{d}$ clearly decreases as a function of $p_{T}\left(H_{b}\right)$. The slope of this dependence is similar to the one measured for the $B_{s}^{0}$ meson. The average production fraction times the $B_{c} \rightarrow J / \psi \mu \nu$ branching fraction, in the interval $4<p_{T}\left(H_{b}\right)<25 \mathrm{GeV} / c$ and for the $13 \mathrm{TeV}$ sample, is

$$
\frac{f_{c}}{f_{u}+f_{d}} \mathscr{B}\left(B_{c}^{-} \rightarrow J / \psi \mu \nu\right)=\left(7.36 \pm 0.08_{\text {stat }} \pm 0.30_{\text {syst }}\right) \times 10^{-5},
$$

where the dominant source of systematic uncertainty is due to the shapes of the signal and background components used to fit the $m_{c o r}$ distribution, which are obtained from the simulation.

The predicted branching fraction of $\mathscr{B}\left(B_{c}^{-} \rightarrow J / \psi \mu \nu\right)$ available in the literature vary from $1.3 \%$ to $2.7 \%$ (see references in [8]). Using as the branching fraction an average value of $1.95 \%$, the $B_{c}^{-}$production fraction ratio at $13 \mathrm{TeV}$ is

$$
\frac{f_{c}}{f_{u}+f_{d}}=\left(3.78 \pm 0.04_{\text {stat }} \pm 0.15_{\text {syst }} \pm 0.89_{B F}\right) \times 10^{-5},
$$

where the third uncertainty, which is $24 \%$, is due to the spread of the theoretical predictions of $\mathscr{B}\left(B_{c}^{-} \rightarrow J / \psi \mu \nu\right)$. The HPQCD collaboration provided a first prediction of the $B_{c}^{-} \rightarrow J / \psi \mu \nu$ decay width [26], which has an uncertainty of only $6.5 \%$.

The first measurement of $f_{c}$ summarized here, allows to extract the absolute branching fractions of the $B_{c}^{-}$meson decays. 

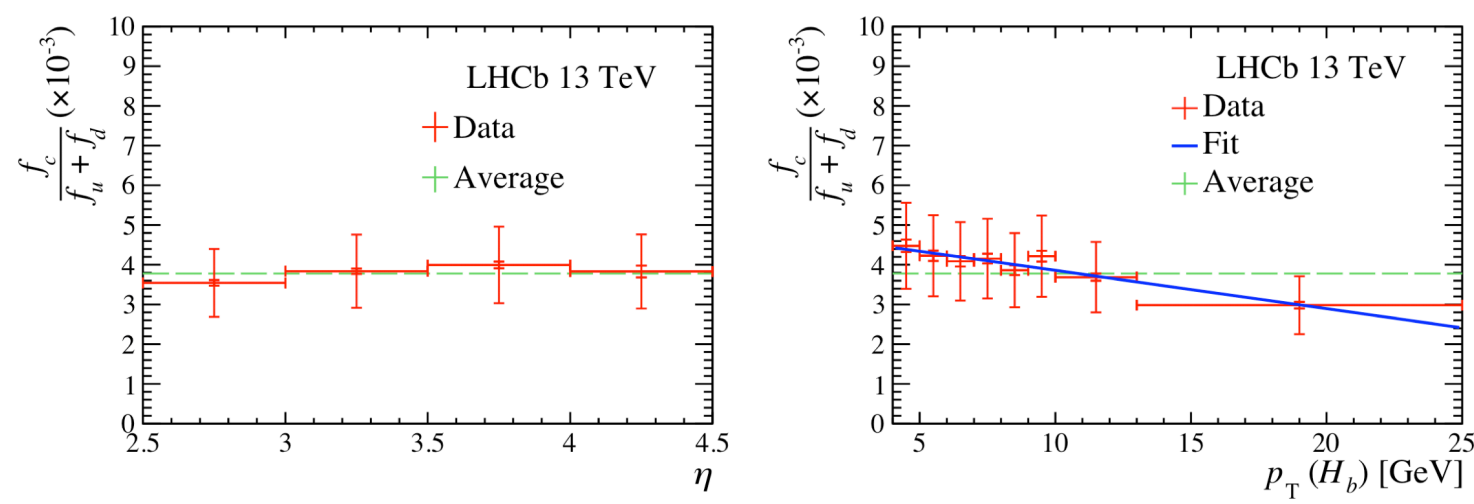

Figure 9: Ratio of production fractions as a function of $\eta$ (left) and $p_{T}\left(H_{b}\right)$ (right) in $13 \mathrm{TeV}$ data.

\section{$3.4 \Xi_{b}$ production at $\sqrt{s}=7,8$ and $13 \mathrm{TeV}$}

Recently LHCb has made some first observations of specific decay modes of various $b$-baryons like $\Xi_{b}^{0}, \Xi_{b}^{+}$and $\Omega_{b}^{+}[27,28,29,30]$. However the absolute branching fractions cannot be accessed because there are no measurements of the $f_{\Xi_{b}^{0}}, f_{\Xi_{b}^{-}}$and $f_{\Omega_{b}^{-}}$fragmentation fractions. The same approach used to extract $f_{s} / f_{d}$ could in principle be used to access $f_{\Xi_{b}}$ using the semileptonic inclusive decays $\Xi_{b}^{0} \rightarrow \Xi_{c}^{+} \mu \nu X$ and $\Xi_{b}^{-} \rightarrow \Xi_{c}^{0} \mu v X$. However the absolute branching fractions for the decays of $\Xi_{c}^{+}$and $\Xi_{c}^{0}$ are not known well, so this approach is not feasible.

The alternative approach employed in this analysis, is to use the decays $\Lambda_{b}^{0} \rightarrow J / \psi \Lambda$ and $\Xi_{b}^{-} \rightarrow$ $J / \psi \Xi^{-}$, with $\Xi^{-} \rightarrow \Lambda \pi^{-}$. Charge-conjugate processes are implicitly included. These processes are related through $S U(3)$ flavor symmetry, $\Gamma\left(\Xi_{b}^{-}\right) / \Gamma\left(\Lambda_{b}^{0}\right)=3 / 2$. The ratio $R$ of the yields of $\Xi_{b}^{-} \rightarrow J / \psi \Xi^{-}$and $\Lambda_{b}^{0} \rightarrow J / \psi \Lambda$, corrected for the detection efficiency, is connected to $f_{\Xi_{b}^{-}} / f_{\Lambda_{b}^{0}}$ through

$$
R=\frac{f_{\Xi_{b}^{-}}}{f_{\Lambda_{b}^{0}}} \frac{\mathscr{B}\left(\Xi_{b}^{-} \rightarrow J \psi \Xi^{-}\right)}{\mathscr{B}\left(\Lambda_{b}^{0} \rightarrow J / \psi \Lambda\right)}=\frac{f_{\Xi_{b}^{-}}}{f_{\Lambda_{b}^{0}}} \frac{\mathscr{B}\left(\Xi_{b}^{-} \rightarrow J \psi \Xi^{-}\right)}{\mathscr{B}\left(\Lambda_{b}^{0} \rightarrow J / \psi \Lambda\right)} \frac{\tau_{\Xi_{b}}}{\tau_{\Lambda_{b}}},
$$

which depends on the assumptions on the ratio of decay widths and the known ratio of lifetimes. The decays $\Xi_{b}^{-} \rightarrow J / \psi \Xi^{-}\left(\Xi^{-} \rightarrow \Lambda \pi^{-}\right)$and $\Lambda_{b}^{0} \rightarrow J / \psi \Lambda$ both have a $J / \psi$ and a $\Lambda$ in the final state, and have very similar kinematics. This feature allows strong cancellations in the detection systematic uncertainties. Because the $\Xi^{-}$and $\Lambda$ have long lifetimes, most of the $\Lambda$ from $\Xi^{-}$do not decay in the vertex detector and are reconstructed only from information from the trackers before and after the dipole magnet. To equalize the selection efficiencies, the $\Lambda$ from $\Lambda_{b}$ decays are also required to decay after the vertex detector. This reduces the yields of the reconstructed $\Lambda_{b}$, but allows a better control of the systematic uncertainties. Using data collected at $\sqrt{s}=7$ and $8 \mathrm{TeV}$ the ratio of production rate is $R=\left(10.8 \pm 0.9_{\text {stat }} \pm 0.8_{\text {syst }}\right) \times 10^{-2}$, and at $\sqrt{s}=13 \mathrm{TeV}$ the ratio is $R=\left(13.1 \pm 1.1_{\text {stat }} \pm 1.0_{\text {syst }}\right) \times 10^{-2}$ where the uncertainties are statistical and experimental. The largest source of uncertainty is the assumption on the polarisations of the $\Lambda_{b}$ and $\Xi_{b}^{-}$at production, and the detection efficiency of the $\pi^{-}$from the $\Xi^{-}$that enters directly into the ratio $R$. The results are obtained in the kinematic range $p_{T}<20 \mathrm{GeV} / c$ and $2<\eta<6$. Assuming $S U$ (3) symmetry, 
the ratio of fragmentation fractions at $13 \mathrm{TeV}$ is

$$
\frac{f_{\Xi_{b}^{-}}}{f_{\Lambda_{b}^{0}}}=\left(8.2 \pm 0.7_{\text {stat }} \pm 0.6_{\text {syst }} \pm 2.5_{S U(3)}\right) \times 10^{-2},
$$

which is consistent with the results at 7 and $8 \mathrm{TeV}$ obtained in the same analysis, $f_{\Xi_{b}^{-}} / f_{\Lambda_{b}^{0}}=$ $\left.6.7 \pm 0.5_{\text {stat }} \pm 0.5_{\text {syst }} \pm 2.0_{S U(3)}\right) \times 10^{-2}$. The last uncertainty of $30 \%$ is the typical size of $S U(3)$ breaking effects. These results are consistent with the predictions of [31]. To summarize, in the forward direction, assuming an equal production of $\Xi_{b}^{-}$and $\Xi_{b}^{0}$, the $b$-quark fragments into $\Xi_{b}$ at a rate of about $15 \%$ of the rate of $\Lambda_{b}$ production.

\section{Conclusions}

Several recent results on heavy flavour production in $p p$ collisions have been reported. In general the differential cross-sections and the ratio of cross-sections at different centre of mass energies are described by predictions within uncertainties. But there are still inconsistencies that require further efforts from both theory and experiment. The precisions on $f_{s}$ and $f_{\Lambda_{b}^{0}}$ production fractions have been greatly improved, this will allow precise measurements of absolute branching fractions of various decay modes. The presented measurement of $f_{c}$ opens various opportunities with $B_{c}$ decays. All the measurements presented provide important input for the tuning of simulations which are needed for precise evaluation of the detector acceptance of many decay processes, for all the LHC experiments.

\section{References}

[1] R. Aaij et al. [LHCb Collaboration], Observation of $J / \psi p$ Resonances Consistent with Pentaquark States in $\Lambda_{b}^{0} \rightarrow J / \psi K^{-} p$ Decays, Phys. Rev. Lett. 115 (2015) 072001 doi:10.1103/PhysRevLett.115.072001 [arXiv:1507.03414 [hep-ex]].

[2] A. Alves, Jr. et al. [LHCb Collaboration], The LHCb Detector at the LHC, JINST 3 (2008), S08005 doi:10.1088/1748-0221/3/08/S08005

[3] R. Aaij et al. [LHCb Collaboration], LHCb Detector Performance, Int. J. Mod. Phys. A 30 (2015) no.07, 1530022 doi:10.1142/S0217751X15300227 [arXiv:1412.6352 [hep-ex]].

[4] R. Aaij et al. [LHCb Collaboration], Measurement of $\psi(2 S)$ production cross-sections in proton-proton collisions at 7 and $13 \mathrm{TeV}$, Eur. Phys. J. C 80 (2020) no.3, 185 doi:10.1140/epjc/s10052-020-7638-y [arXiv:1908.03099 [hep-ex]].

[5] R. Aaij et al. [LHCb Collaboration], Measurement of the $\eta_{c}(1 S)$ production cross-section in $p p$ collisions at $\sqrt{s}=13 \mathrm{TeV}$, Eur. Phys. J. C 80 (2020) no.3, 191 doi:10.1140/epjc/s10052-020-7733-0 [arXiv:1911.03326 [hep-ex]].

[6] R. Aaij et al. [LHCb Collaboration], Measurement of $\Xi_{c c}^{++}$production in pp collisions at $\sqrt{s}=13$ TeV, Chin. Phys. C 44 (2020) no.2, 022001 doi:10.1088/1674-1137/44/2/022001 [arXiv:1910.11316 [hep-ex]].

[7] R. Aaij et al. [LHCb Collaboration], Measurement of b hadron fractions in $13 \mathrm{TeV}$ pp collisions, Phys. Rev. D 100 (2019) no.3, 031102 doi:10.1103/PhysRevD.100.031102 [arXiv:1902.06794 [hep-ex]]. 
[8] R. Aaij et al. [LHCb Collaboration], Measurement of the $B_{c}^{-}$meson production fraction and asymmetry in 7 and 13 TeV pp collisions, Phys. Rev. D 100 (2019) no.11, 112006 doi:10.1103/PhysRevD.100.112006 [arXiv:1910.13404 [hep-ex]].

[9] R. Aaij et al. [LHCb Collaboration], Measurement of the mass and production rate of $\Xi_{b}^{-}$baryons, Phys. Rev. D 99 (2019) no.5, 052006 doi:10.1103/PhysRevD.99.052006 [arXiv:1901.07075 [hep-ex]].

[10] G. T. Bodwin, E. Braaten and G. P. Lepage, Rigorous QCD analysis of inclusive annihilation and production of heavy quarkonium, Phys. Rev. D 51 (1995) 1125 Erratum: [Phys. Rev. D 55 (1997) 5853] doi:10.1103/PhysRevD.55.5853, 10.1103/PhysRevD.51.1125 [hep-ph/9407339]. P. L. Cho and A. K. Leibovich, Color octet quarkonia production, Phys. Rev. D 53 (1996) 150 doi:10.1103/PhysRevD.53.150 [hep-ph/9505329].

[11] M. Cacciari, M. Greco and P. Nason, The $p_{T}$ spectrum in heavy flavor hadroproduction, JHEP 9805 (1998) 007 doi:10.1088/1126-6708/1998/05/007 [hep-ph/9803400].

[12] R. Aaij et al. [LHCb Collaboration], Measurement of forward J/ $\psi$ production cross-sections in $p p$ collisions at $\sqrt{s}=13 \mathrm{TeV}$, JHEP 1510 (2015) 172 Erratum: [JHEP 1705 (2017) 063] doi:10.1007/JHEP05(2017)063, 10.1007/JHEP10(2015)172 [arXiv:1509.00771 [hep-ex]].

[13] R. Aaij et al. [LHCb Collaboration], Measurement of the $\eta_{c}(1 S)$ production cross-section in proton-proton collisions via the decay $\eta_{c}(1 S) \rightarrow p \bar{p}$, Eur. Phys. J. C 75 (2015) no.7, 311 doi:10.1140/epjc/s10052-015-3502-x [arXiv:1409.3612 [hep-ex]].

[14] Y. Feng, J. He, J. P. Lansberg, H. S. Shao, A. Usachov and H. F. Zhang, Phenomenological NLO analysis of $\eta_{c}$ production at the LHC in the collider and fixed-target modes, Nucl. Phys. B 945 (2019) 114662 doi:10.1016/j.nuclphysb.2019.114662 [arXiv:1901.09766 [hep-ph]].

[15] M. Butenschoen, Z. G. He and B. A. Kniehl, $\eta_{c}$ production at the LHC challenges nonrelativistic-QCD factorization, Phys. Rev. Lett. 114 (2015) no.9, 092004 doi:10.1103/PhysRevLett.114.092004 [arXiv:1411.5287 [hep-ph]].

[16] H. Han, Y. Q. Ma, C. Meng, H. S. Shao and K. T. Chao, $\eta_{c}$ production at LHC and indications on the understanding of J/ $\psi$ production, Phys. Rev. Lett. 114 (2015) no.9, 092005 doi:10.1103/PhysRevLett.114.092005 [arXiv:1411.7350 [hep-ph]].

[17] H. F. Zhang, Z. Sun, W. L. Sang and R. Li, Impact of $\eta_{c}$ hadroproduction data on charmonium production and polarization within NRQCD framework, Phys. Rev. Lett. 114 (2015) no.9, 092006 doi:10.1103/PhysRevLett.114.092006 [arXiv:1412.0508 [hep-ph]].

[18] R. Aaij et al. [LHCb Collaboration], Observation of the doubly charmed baryon $\Xi_{c c}^{++}$, Phys. Rev. Lett. 119 (2017) no.11, 112001 doi:10.1103/PhysRevLett.119.112001 [arXiv:1707.01621 [hep-ex]].

[19] R. Aaij et al. [LHCb Collaboration], Measurement of the Lifetime of the Doubly Charmed Baryon $\Xi_{c c}^{++}$, Phys. Rev. Lett. 121 (2018) no.5, 052002 doi:10.1103/PhysRevLett.121.052002 [arXiv:1806.02744 [hep-ex]].

[20] I. I. Bigi, T. Mannel and N. Uraltsev, Semileptonic width ratios among beauty hadrons, JHEP 1109 (2011) 012 doi:10.1007/JHEP09(2011)012 [arXiv:1105.4574 [hep-ph]].

[21] M. Tanabashi et al. [Particle Data Group], Review of Particle Physics Phys. Rev. D 98 (2018) no.3, 030001. doi:10.1103/PhysRevD.98.030001

[22] R. Aaij et al. [LHCb Collaboration], Measurement of b-hadron production fractions in $7 \mathrm{TeV} p p$ collisions, Phys. Rev. D 85 (2012), 032008 doi:10.1103/PhysRevD.85.032008 [arXiv:1111.2357 [hep-ex]]. 
[23] R. Aaij et al. [LHCb Collaboration], Study of the kinematic dependences of $\Lambda_{b}^{0}$ production in pp collisions and a measurement of the $\Lambda_{b}^{0} \rightarrow \Lambda_{c}^{+} \pi^{-}$branching fraction, JHEP 08 (2014), 143 doi:10.1007/JHEP08(2014)143 [arXiv:1405.6842 [hep-ex]].

[24] R. Aaij et al. [LHCb], Measurement of $f_{s} / f_{u}$ variation with proton-proton collision energy and B-meson kinematics, Phys. Rev. Lett. 124 (2020) no.12, 122002 doi:10.1103/PhysRevLett.124.122002 [arXiv:1910.09934 [hep-ex]].

[25] R. Aaij et al. [LHCb Collaboration], Measurement of the fragmentation fraction ratio $f_{s} / f_{d}$ and its dependence on B meson kinematics, JHEP 1304 (2013) 001 doi:10.1007/JHEP04(2013)001 [arXiv:1301.5286 [hep-ex]]; $f_{s} / f_{d}$ value updated in LHCb-CONF-2013-011.

[26] J. Harrison, $B_{c} \rightarrow J / \psi$ Form Factors and $R(J / \psi)$ using Lattice $Q C D$, preliminary results at this conference.

[27] R. Aaij et al. [LHCb Collaboration], Studies of beauty baryon decays to $D^{0} p h^{-}$and $\Lambda_{c}^{+} h^{-}$final states, Phys. Rev. D 89 (2014) no.3, 032001 doi:10.1103/PhysRevD.89.032001 [arXiv:1311.4823 [hep-ex]].

[28] R. Aaij et al. [LHCb Collaboration], Evidence for the strangeness-changing weak decay $\Xi_{b}^{-} \rightarrow \Lambda_{b}^{0} \pi^{-}$, Phys. Rev. Lett. 115 (2015) no.24, 241801 doi:10.1103/PhysRevLett.115.241801 [arXiv:1510.03829 [hep-ex]].

[29] R. Aaij et al. [LHCb Collaboration], Precision measurement of the mass and lifetime of the $\Xi_{b}^{0}$ baryon, Phys. Rev. Lett. 113 (2014), 032001 doi:10.1103/PhysRevLett.113.032001 [arXiv:1405.7223 [hep-ex]].

[30] R. Aaij et al. [LHCb Collaboration], Observation of the $\Xi_{b}^{-} \rightarrow J / \psi \Lambda K^{-}$decay, Phys. Lett. B 772 (2017), 265-273 doi:10.1016/j.physletb.2017.06.045 [arXiv:1701.05274 [hep-ex]].

[31] H. Y. Jiang and F. S. Yu, Fragmentation-fraction ratio $f_{\Xi_{b}} / f_{\Lambda_{b}}$ in b-and c-baryon decays, Eur. Phys. J. C 78 (2018) no.3, 224 doi:10.1140/epjc/s10052-018-5704-5 [arXiv:1802.02948 [hep-ph]]. 